\title{
Correction to: Pathway crosstalk perturbation network modeling for identification of connectivity changes induced by diabetic neuropathy and pioglitazone
}

Guillermo de Anda-Jáuregui ${ }^{1,3}$, Kai Guo ${ }^{1}$, Brett A. McGregor ${ }^{1}$, Eva L. Feldman² and Junguk Hur ${ }^{1 *}$

\section{Correction to: BMC Systems Biology 20149 13:1 https://doi.org/10.1186/s12918-018-0674-7}

Following publication of the original article [1], it was highlighted that the funding statement was not correct. This Correction article shows the correct and incorrect Funding section.

Correct:

The National Institute of Diabetes and Digestive and Kidney Diseases (NIDDK; 1R24082841 to E.L.F. and J.H.), the NIDDK Diabetic Complications Consortium Pilot Grant (DiaComp, https://diacomp.org/; DK076169; sub-award \#25034-75 to J.H.), and University of North Dakota Post-Doc Pilot Grant (to K.G.), and the North Dakota EPSCoR through National Science Foundation grant \#OIA-1355466 (to B.A.M.). Funding bodies had no role in the design, execution, or analysis of this work.

Incorrect:

The National Institute of Diabetes and Digestive and Kidney Diseases (NIDDK; 1R24082841 to E.L.F. and J.H.), the NIDDK Diabetic Complications Consortium Pilot Grant (DiaComp, https://diacomp.org/; DK076169; subaward \#25034-75 to J.H.), and University of North Dakota Post-Doc Pilot Grant (to K.G.). Funding bodies had no role in the design, execution, or analysis of this work.

\section{Author details}

'Department of Biomedical Sciences, University of North Dakota School of Medicine and Health Sciences, Grand Forks, North Dakota 58202, USA.

${ }^{2}$ Department of Neurology, University of Michigan School of Medicine, Ann Arbor, MI 48109, USA. ${ }^{3}$ Present address: Computational Genomics Division, Instituto Nacional de Medicina Genómica, 14610 Ciudad de México, Mexico.
Received: 8 March 2019 Accepted: 8 March 2019 Published online: 13 March 2019

\section{Reference}

1. de Anda-Jáuregui $G$, et al. Pathway crosstalk perturbation network modeling for identification of connectivity changes induced by diabetic neuropathy and pioglitazone. BMC Syst Biol. 2019;13(1). https://doi.org/10.1186/s12918-018-0674-7.

\footnotetext{
* Correspondence: junguk.hur@med.und.edu

'Department of Biomedical Sciences, University of North Dakota School of Medicine and Health Sciences, Grand Forks, North Dakota 58202, USA Full list of author information is available at the end of the article
}

(c) The Author(s). 2019 Open Access This article is distributed under the terms of the Creative Commons Attribution 4.0 International License (http://creativecommons.org/licenses/by/4.0/), which permits unrestricted use, distribution, and reproduction in any medium, provided you give appropriate credit to the original author(s) and the source, provide a link to the Creative Commons license, and indicate if changes were made. The Creative Commons Public Domain Dedication waiver (http://creativecommons.org/publicdomain/zero/1.0/) applies to the data made available in this article, unless otherwise stated. 